F. Grimminger

D. Walmrath

\section{Lungenprotektive Beatmung verringert ARDS-Mortalität}

\author{
Ergebnisse einer prospektiven, randomisierten, \\ multizentrischen Studie der ARDS-Network Study Group \\ in Nordamerika
}

Das Syndrom des akuten hypoxämischen Lungenversagens (acute respiratory distress syndrome/ARDS) ist charakterisiert durch eine akute Beeinträchtigung des Gasaustausches und beruht pathophysiologisch auf einer entzündungsbedingten Mikrozirkulationsstörung mit Ausbildung eines interstitiellen und alveolären Ödems sowie einer alveolären Instabilität mit diffuser Mikroatelektasenbildung. Das Lungenparenchym ist dabei nicht homogen betroffen, sondern zerfällt in drei funktionelle Kompartimente:

- Alveolarbezirke, die aufgrund der ödembedingten Surfactantstörung komplett kollabiert sind und nur durch hohe Druckmanöver rekrutiert werden können,

- Alveolarbezirke, die zyklisch endexspiratorisch kollabieren und inspiratorisch reexpandiert werden,

- Stabile Alveolarbezirke (gesunde Restlunge).

Die invasive Beatmung dient der Überbrückung des lebensbedrohlichen hypoxämischen Zustandes und ist in ihrem Stellenwert nach wie vor unumstritten. Doch mussten wir in den letzten Jahren auch lernen, dass die Beatmung selbst ein Trauma für das Lungenparenchym darstellt und ein bestehendes ARDS nicht nur protrahieren sondern gar aggravieren kann. In tierexperimentellen Untersuchungen ließ sich durch bestimmte Atmungsformen ein ARDS in einem vorher gesunden Or-

Prof. Dr. F. Grimminger ( ) PD Dr. D. Walmrath

Medizinische Klinik II

des Zentrums für Innere Medizin

der Justus-Liebig-Universität Gießen

D-35423 Gießen gan induzieren. Dieses Phänomen beruht auf der Anwendung undifferenzierter, invasiver Beatmungsschemata, deren Effizienz allein an den Parametern des Gasaustausches nicht aber an den unterschiedlichen mechanischen Charakteristika der 3 Kompartimente orientiert sind. Vor diesem Hintergrund wurde postuliert, dass Beatmungsstrategien, welche die drei Kompartimenttheorie der ARDS-Lunge berücksichtigen, mit einer signifikanten Reduktion der Letalität einhergehen könnten und das Konzept der protektiven Beatmung entwickelt, welches der nunmehr veröffentlichten Studie der ARDSNetwork Study Group zu Grunde liegt.

\section{Mechanismen der beatmungsinduzierten Lungenschädigung - protektive Beatmung}

In den drei genannten Kompartimenten der ARDS-Lunge kommen unterschiedliche Schädigungsmechanismen zum Tragen:

1. In den atelektatischen Alveolarbezirken kann durch entsprechende Spitzendrucke eine inspiratorische Reexpansion mit zyklischem exspiratorischem Kollaps als schädigender Faktor für die knickungssensiblen Alveolarsepten auftreten. Das Konzept der lungenprotektiven Beatmung beinhaltet die Ruhigstellung dieser druckrekrutierbaren Bezirke durch Begrenzung der Spitzendrucke.

2. Ein durch das Beatmungsgerät verabreichter Atemgasfluss folgt dem Weg des geringsten Widerstandes durch die Lunge und wird deshalb bevorzugt in das kleine Kompartiment der gesunden Restlunge mit normaler Compliance zu Ungunsten der surfactantgeschädigten instabilen Kompartimente gelangen. Das vermutete Mechanotrauma beruht auf einer Überextension dieser Alveolen. Diesem Schädigungsmechanismus kann durch eine Begrenzung des Hubvolumens begegnet werden. 
3. Kontrovers diskutiert wird gegenwärtig, welche PEEPEinstellung für die Minimierung von Lungenschäden unter der Beatmung am günstigsten ist: Ein ausreichend hoher PEEP kann in dem Lungenkompartiment mit instabilen endexspiratorisch kollabierenden Alveolen eine zyklische Traumatisierung durch Expansion und Reokklusion verhindern. Ein zu hoher PEEP kann andererseits in den Lungenarealen mit normaler Compliance zum Mechanismus des Baro- bzw. Volutraumas beitragen. Angesichts der Unklarheit, ob der Mechanismus der Überexpansion bzw. des zyklischen Alveolarkollaps pathophysiologisch führend ist, erklärt sich die gegenwärtige Unsicherheit im Hinblick auf den Best-PEEP. Sehr viel pathophysiologische Plausibilität spricht für die Zielvorgabe, durch Auswahl eines PEEP-Wertes oberhalb eines unteren Flexionspunktes möglichst viele Alveolarbezirke zu rekrutieren und vor erneuter Atelektasenbildung bei der nachfolgenden Ausatmung zu schützen. Durch Erstellung einer Druckvolumenschleife kann ein solcher Druckwert zumeist definiert werden. Dieses Vorgehen erlaubt auch die Charakterisierung eines oberen Flexionspunktes, bei dem offensichtlich eine Überdehnung von Lungenparenchymstrukturen in größerem Umfang beginnt.

\section{Klinische Erfahrung mit protektiver Beatmung - state of the art}

Die Begrenzung der Drucke während des Beatmungszyklus auf den Bereich zwischen den beiden Flexionspunkten ist als Behandlungsprinzip bislang nicht in kontrollierten Studien abgesichert. Eine kürzlich publizierte Untersuchung von Amato (1) beschreibt zwar eine verminderte Letalität von ARDS-Patienten durch Beatmung mit PEEP-Werten oberhalb des zuvor definierten Pflex-unten, jedoch wurden in dieser Studie zugleich das Atemzugvolumen reduziert, es kamen zusätzliche Rekrutierungsmanöver zur Anwendung und die Letalität in der Kontrollgruppe mit konventioneller Beatmung war im internationalen Vergleich ungewöhnlich hoch. Andere zeitgleich durchgeführte prospektiv randomisierte Studien (2-4) kamen zu widersprüchlichen Ergebnissen, wobei die Vergleichbarkeit der Ergebnisse dadurch erschwert wird, dass jeweils gegenüber den Kontrollgruppen unterschiedliche Parameter der Beatmungstherapie in der Studiengruppe variiert wurden (Spitzendruck, Zugvolumen, permissive Hyperkapnie, Höhe des PEEP).

In der nun vorliegenden Studie der ARDS-Network Group (5) wurde gegenüber der Kontrollgruppe mit dem konventionellen Beatmungsprotokoll lediglich das Zugvolumen mit $6 \mathrm{ml} / \mathrm{kg}$ Körpergewicht gegenüber $12 \mathrm{ml} / \mathrm{kg}$ Körpergewicht variiert. Der PEEP wurde in beiden Gruppen nach einem Algorithmus eingestellt. Über- lagernde Effekte durch das Prinzip der permissiven Hyperkapnie wurden ausgeschlossen, indem in der Studiengruppe, die aufgrund des niedrigen Zugvolumens zu Hyperkapnie neigte, konsequent die respiratorische Azidose mit Infusion von Bicarbonat ausgeglichen wurde bzw. durch Erhöhung der Atemfrequenz. Die Studie wurde gestoppt als eine Interimsanalyse eine Abnahme der Mortalität in der Studiengruppe um 22\% dokumentierte. Die Zurücknahme des Zugvolumens kann ebenso zu einer Abnahme der arteriellen Oxygenierung führen. Das traditionelle Ziel der mechanischen Beatmung war es, einen arteriellen $\mathrm{PO}_{2}$ von mind. $60 \mathrm{mmHg}$ oder einer Hämoglobinsättigung von $90 \%$ zu erreichen. Ein Ziel, das oft durch die Eskalierung der Beatmungsdrucke und erst dann durch eine Anhebung der inspiratorischen Sauerstoffkonzentration erreicht wurde. Interessanterweise erlaubt die ARDS-Network Group in dieser Studie deutlich niedrigere Werte $\left(\mathrm{PO}_{2} 55 \mathrm{mmHg}\right.$, Hämoglobinsättigung 88\%). Fazit ist, dass bei ARDS-Patienten das Atemzugvolumen zur Verbesserung der Überlebensprognose deutlich reduziert werden muss, mit dem Ziel, einen Plateaudruck von unter $32 \mathrm{~cm}$ Wassersäule zu erreichen selbst um den Preis eines zeitweise nicht optimierten Gasaustausches bei bewusst unvollständiger Ausschöpfung der Möglichkeiten des Respirators. Die Frage, ob nicht gar noch niedrigere Plateaudrücke angestrebt werden sollten, müssen zukünftige Studien klären.

\section{Schlussfolgerung}

Die Ergebnisse dieser Studie belegen eindrucksvoll, dass eine sanfte Beatmung mit niedrigen Zugvolumina ein Überleben auf der Intensivstation wahrscheinlicher macht. Die Senkung der Mortalität ist um so erstaunlicher als über drei Jahrzehnte hin mit einem erheblichen Aufwand versucht wurde, eine Mortalitätsabnahme mit pharmakologischen Mitteln (Glukokortikoide, Prostazyklin, Stickstoffmonoxyd, Antizytokine) zu erreichen, ohne dass jemals über einen gelegentlich beobachteten Akutbenefit hinaus ein signifikanter Effekt auf die 28-Tage-Mortalität unstrittig bestätigt werden konnte. Die Behandlung der Lunge scheint ein prognostisch entscheidender Faktor zu sein und es kann erwartet werden, dass eine weitere Outcome-Optimierung durch die Definition eines individuell titrierten Best-PEEP, eine Festlegung auf Hyperkapnie-Toleranzgrenzen und eine Optimierung der Exspirations-Inspirations-Verhältnisse die erstaunlichen Resultate dieser Studie noch weiter verbessern können. Eine noch weitreichendere Reduktion des Beatmungstraumas gelingt möglicherweise in Zukunft durch die Kombination von protektiven Beatmungsregimen mit der Gabe von exogenem Surfactant und inhalativen Vasodilatoren zur pharmakologischen Optimierung des Gasaustausches. 


\section{Literatur}

1. Amato MB, Barbas CS, Medeiros DM, Magaldi RB, Schettino GP, Lorenzi-Filho G, Kairalla RA, Deheinzelin D, Munoz C, Oliveira R, Takagaki TY, Carvalho CR (1998) Effect of a protective-ventilation strategy on mortality in the acute respiratory distress syndrome. N Engl J Med 338:347-354

2. Brochard L, Roudot-Thoraval F, Roupie E, Delclaux C, Chastre J, Fernandez-Mondejar E, Clementi E, Mancebo J, Factor P, Matamis D, Ranieri M, Blanch L, Rodi G, Mentec H, Dreyfuss D, Ferrer M, Brun-Buisson $\mathrm{C}$, Tobin $\mathrm{M}$, Lemaire $\mathrm{F}$ (1998) Tidal volume reduction for prevention of ventilator-induced lung injury in acute respiratory distress syndrome. The Multicenter Trial Group on Tidal Volume reduction in ARDS. Am J Respir Crit Care Med 158:1831-1838
3. Stewart TE, Meade MO, Cook DJ, Granton JT, Hodder RV, Lapinsky SE, Mazer CD, McLean RF, Rogovein TS, Schouten BD, Todd TR, Slutsky AS (1998) Evaluation of a ventilation strategy to prevent barotrauma in patients at high risk for acute respiratory distress syndrome. Pressure- and Volume-Limited Ventilation Strategy Group. N Engl J Med 338:355361

4. Brower RG, Shanholtz CB, Fessler HE, Shade DM, White P Jr, Wiener CM, Teeter JG, Dodd-o JM, Almog Y, Piantadosi S (1999) Prospective, randomized, controlled clinical trial comparing traditional versus reduced tidal volume ventilation in acute respiratory distress syndrome patients. Crit Care Med 27:1492-1498
5. The Acute Respiratory Distress Syndrome Network (2000) Ventilation with lower tidal volumes as compared with traditional tidal volumes for acute lung injury and the acute respiratory distress syndrome. $\mathrm{N}$ Engl J Med 342:1301-1308 